\title{
A Long-Term Diabetic Autonomic Nervous Abnormality
}

\author{
Reduced Variations in Resting Heart Rate Measured by a Simple and Sensitive Method
}

\author{
H. J. G. Gundersen and B. Neubauer \\ 2nd University Clinic of Internal Medicine, University of Aarhus, Denmark
}

\begin{abstract}
Summary. Three types of variation in resting pulse rate were measured, using simple and reproducible methods, in 44 young juvenile diabetics and 11 controls. Diabetics with few years duration of the disease showed normal values of both long-term and cyclic fluctuations, as well as of non-systematic, random variation from beat-to-beat. All three types of variation were reduced in long-term diabetics, and the reduction was in all cases correlated with the duration of diabetes. The decrease in non-systematic, beat-to-beat variation attained statistical significance after 5 to 10 years duration of diabetes. It is concluded that reduced variation in resting pulse rate is a long-term diabetic manifestation and that the decrease of non-systematic, beat-to-beat variation in diabetics is a useful indicator of functional abnormalities of the autonomic nervous system.
\end{abstract}

Key words: Long-term diabetes, autonomic nervous system, cardiac denervation, beat-to-beat variation, pulse rate variation, $R R$ interval variation, respiratory arrhythmia, spontaneous variation.

Functional abnormalities of the autonomic nervous system in long-term diabetes are well known [1]. They include postural fall in blood pressure [2], abnormal reflex bradycardia after a Valsalva manoeuvre [3], reduced levels of catecholamines in blood [4] and cardiovascular tissue [5], and a reduction in spontaneous movements of the pupil of the eye [6]. Unfortunately, measures of these abnormalities are either not easily obtained, or are difficult to interpret.

Recently, the exaggerated excursion in heart rate provoked by deep respiration has been shown to be reduced in diabetics with severe neuropathy [7]. A reduction of the variation in the heart rate during standing has also been demonstrated in a selected group of diabetics without clinical signs of autonomic neuropathy [8].

The aim of the present investigation was to study the variations in heart rate by simpler methods and under basal and reproducible conditions. Three types of variation in the heart rate were determined by appropriate statistical methods. The main findings were that all kinds of variation in heart rate were reduced in long-term diabetics and that the severity of these abnormalities correlated well with the duration of diabetes.

\section{Patients and Methods}

Forty-four juvenile, insulin treated diabetics, and eleven non-diabetics (staff-personnel) were studied, all aged between thirty and forty years. Males and females were equally represented in both groups. None had diastolic blood pressure above $100 \mathrm{~mm}$ $\mathrm{Hg}$, an arrhythmia, clinical signs of cardiac disease or ECG-abnormalities. All the diabetics were outpatients and they were unselected with regard to the presence and severity of diabetic complications.

On each subject an ECG was obtained using an ordinary ECG apparatus, and a paper speed of $50 \mathrm{~mm}$ per sec. During this procedure the subjects, who had been at rest for half an hour, were recumbent, breathing spontaneously. They were undisturbed and were unaware when the ECG was recorded. The length in $\mathrm{mm}$ of at least 150 consecutive RR intervals was measured on each ECG using a graphical to digital converter (D-MAC pencil follower), with an accuracy of $0.1 \mathrm{~mm}$.

Three types of pulse rate variation can be distin- 

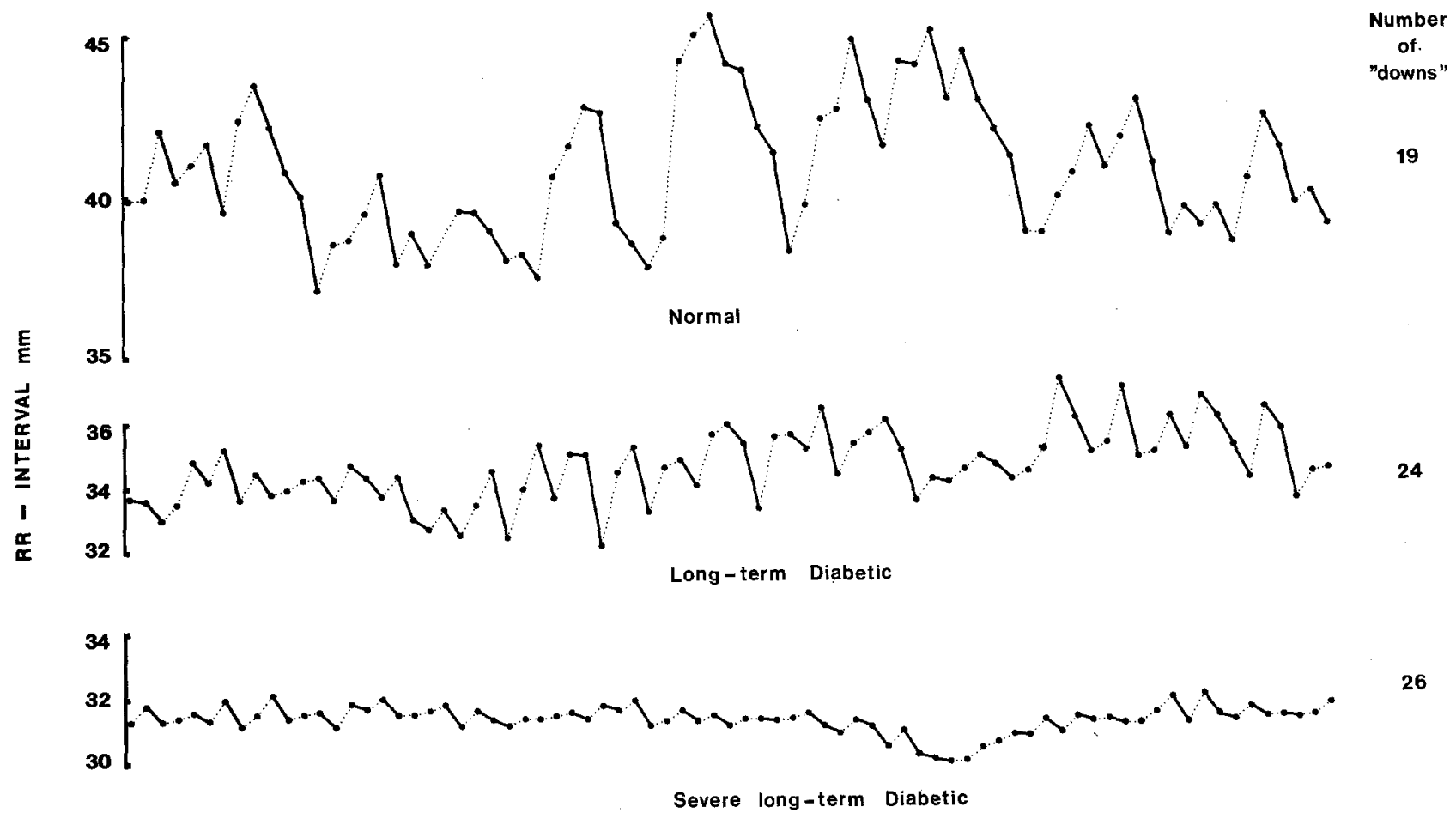

26

Fig. 1. The length of 75 consecutive RR intervals in $\mathrm{mm}$ are given on the ordinate for a normal subject in the middle of the normal range of variation (upper curve) and a median long-term diabetic (middle curve). The lower curve shows the RR intervals from the diabetic in whom least variation was found. The dotted and the full drawn lines indicate runs up and runs down, respectively

guished in the series of RR intervals from a normal subject, an example of which is shown in Figure 1. From beat-to-beat there are some small variations. More obvious, however, is the well-known respiratory arrhythmia appearing as periodic, cyclic oscillations over five to six beats. Furthermore, it is often possible to see shifts or long-term fluctuations of a duration of ten or more beats. The three types of variation in pulse rate were measured in each ECG by three different statistical methods:

1. The mean square successive difference (MSSD), i.e. the standard deviation of the differences between any RR interval and the next [9]. This figure is exclusively a measure of the variation from one beat to the next.

2. The usual standard deviation (SD) of the RR intervals. This is more sensitive to long-term than to short-term fluctuations. If trends or long-term fluctuations are present in a series of RR-intervals the SD is enhanced, whereas the MSSD is almost unaffected.

3. The number of runs-up and runs-down. A run-up is a series of continuously increasing $R R$ intervals, and vice versa. Cyclic variation of the RR interval will reduce the number of runs in the series of consecutive RR intervals. The presence of cyclic influences can therefore be tested by counting the number of runs-up-and-down in the series and comparing (at a 5 per cent level) this figure with the expected number in a random series [9] (see Fig. 1).

\section{Statistical Procedures}

The values of the SD and the MSSD of RR intervals are approximately log-normally distributed within groups of subjects. Accordingly, the differences between groups are tested by means of Student's t-test carried out on the logarithmic transformed values. The mean and the standard deviation of these values are directly related (through the logarithmic function) to the geometric mean and its tolerance factor of the untransformed values. The geometric mean and its tolerance factor are given in the text.

The strength of the relationship between the duration of diabetes and any of the three parameters of variation was measured by Kendall's $\tau$, calculated for $\mathrm{n} \times \mathrm{m}$ tables [10].

\section{Results}

The individual values of the MSSD of RR intervals are shown in Figure 2. The geometric mean was $2.16 \mathrm{~mm} \times / \div 1.41$ in normal subjects, and $1.97 \mathrm{~mm}$ $\mathrm{x} / \div 1.40$ in four short-term diabetics (duration $<5$ years). The difference is not statistically significant $(2 p=0.64)$. The correlation between the MSSD and the duration of diabetes is highly significant (Kendall's $\tau=-0.34 ; 2 \mathrm{p}=0.0078$ ). The geometric mean MSSD of $1.39 \mathrm{~mm} \times / \div 1.48$ in the 5 diabetics with 5 to 10 years duration was significantly lower than that of the controls $(2 p=0.039)$, as 


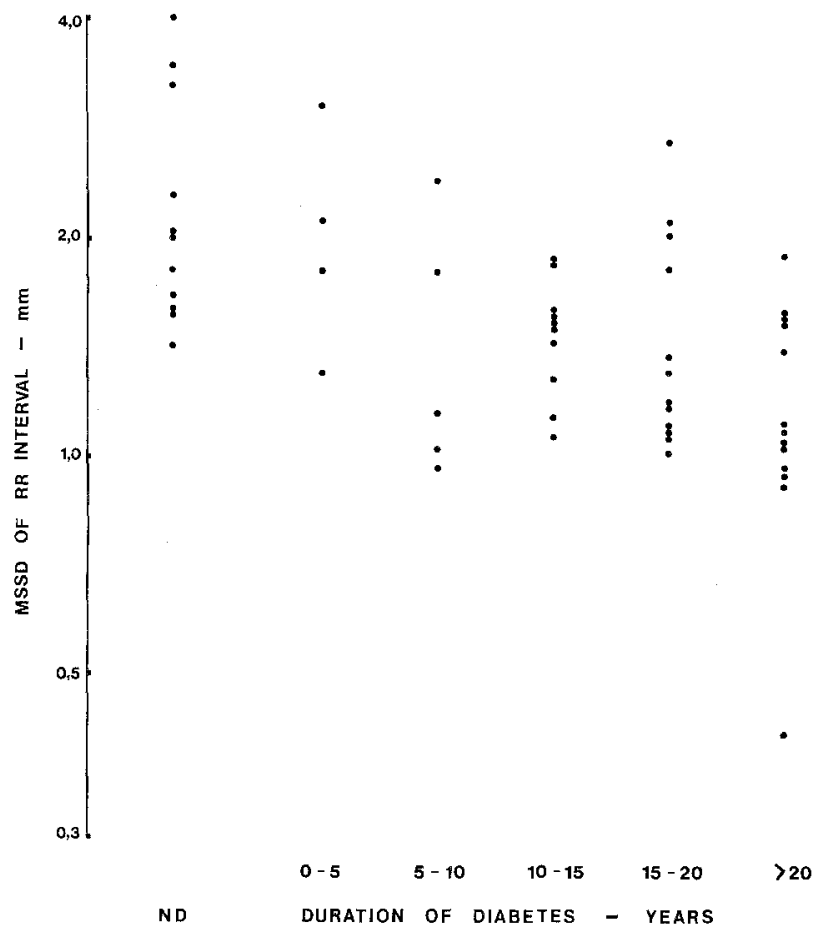

Fig. 2. The individual mean square successive difference (MSSD) of RR intervals in normal subjects (ND) and diabetics. The ordinate is logarithmic

were the geometric means of all groups of diabetics with a longer duration of the disease.

Figure 3 presents the SD of the RR intervals, the parameter especially sensitive to prolonged variations and trends in pulse rate level. The geometric mean SD was $2.08 \mathrm{~mm}^{\mathrm{x}} / \div 1.41$ and $1.98 \mathrm{~mm}^{\mathrm{x}} / \div$ 1.23 in normal subjects and in short-term diabetics, respectively. The difference is not statistically significant $(2 p=0.66)$. The coefficient of correlation between the SD and the duration of diabetes is -0.30 (Kendall's $\tau, 2 p=0.020$ ). The geometric mean SD was $1.46 \mathrm{~mm} x / \div 1.33$ in diabetics with 5 to 10 years duration, which, compared to normals, almost reached statistical significance $(2 p=0.066)$. The groups of diabetics with longer duration all showed decreased SD, that of long-term diabetics (duration $\geqq 20$ years) was half that of the normals: $1.14 \mathrm{~mm}^{\mathrm{x}} / \div 1.56 ; 2 \mathrm{p}=0.0014$

Figure 4 illustrates the results of the test for the expected number of runs-up-and-down. A reduction was found in all normal subjects, indicating the significant cyclic influence of the respiration. The same was found in the four short-term diabetics. The number of subjects in whom no cyclic influence was found increased with the duration of diabetes (Kendall's $\tau=0.34 ; 2 \mathrm{p}=0.012$ ). Sixty per cent of the long-term diabetics had no systematic variation at all in their series of RR intervals, which is significantly different from the proportion in the control group (Fisher's exact test; $2 \mathrm{p}=0.0017$ ).

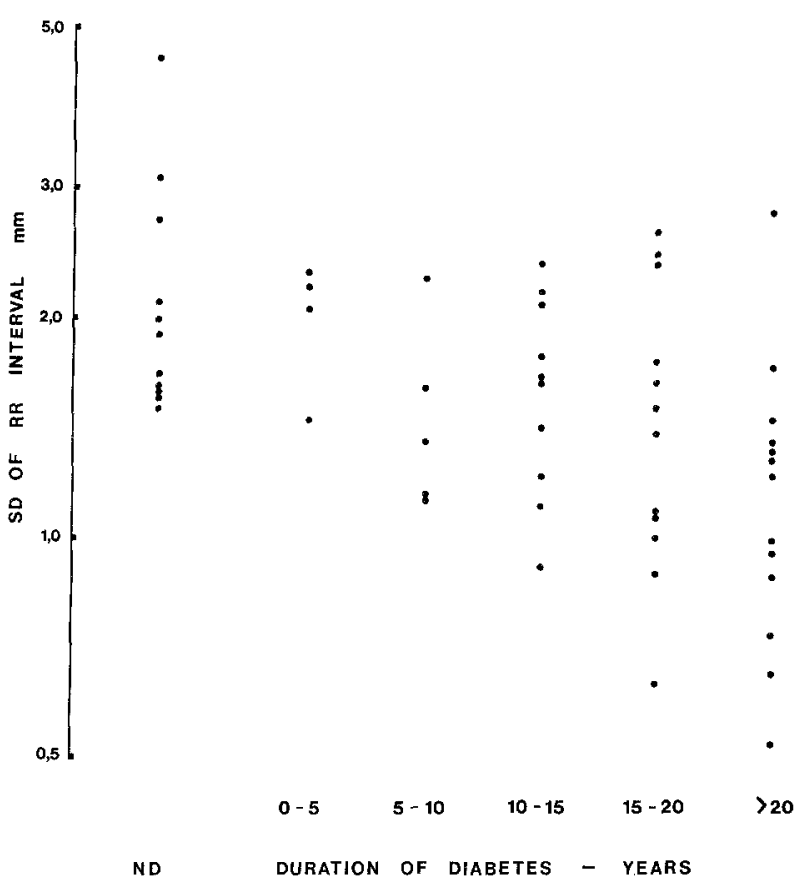

Fig. 3. The standard deviation (SD) of RR intervals in normal subjects (ND) and diabetics. The ordinate is logarithmic

RR INTERVAL FLUCTUATIONS

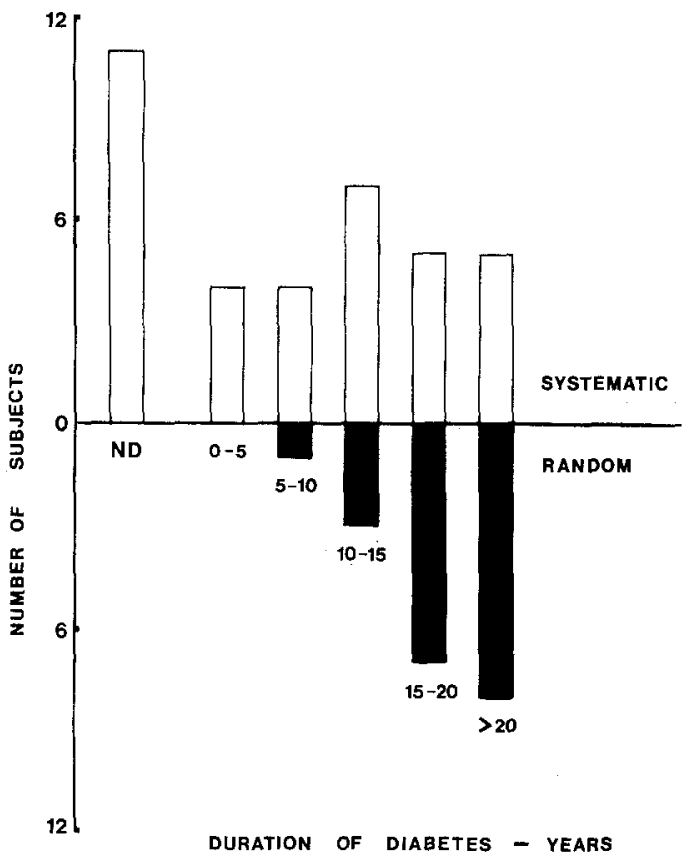

Fig. 4. The white columns show the number of subjects in each group in whom a reduction in the number of runs-up-and-down in the sequence of RR intervals was found. The black columns show the number of subjects in whom such a reduction was not detectable 
There were no significant differences between men and women within any group of subjects.

\section{Discussion}

The results of this study clearly establish that reduction in the variation in resting pulse rate is associated with long-term diabetes. It is not found at the onset of the diabetes and its prevalence increases in relation to the duration of the diabetes.

The fact that all three types of pulse rate variation were found to be abnormal in long-term diabetics indicates that the efferent pathway of the reflex arc is affected.

Wheeler and Watkins measured the difference between maximum and minimum heart rate during deep breathing [7]. They found that this difference was reduced in a group of diabetics with severe autonomic neuropathy. In their experiments the use of deep breathing could imply that the abnormality observed was of purely afferent type.

The standard deviation of RR intervals in diabetics has previously been measured [8]. Due to the experimental design the results, including reduced standard deviation of RR intervals without any correlation to the duration of diabetes in the range of five to twenty-seven years, are difficult to interpret. The study included forty-two diabetics with a minimum of long-term diabetic complications, probably selected in order to form a group fit for exercise testing. None of the patients had a diastolic blood pressure above ninety $\mathrm{mm} \mathrm{Hg}$, none had abnormalities in their exercise ECG and the knee and ankle jerks were absent in only two subjects. Furthermore, the patients were examined at the end of an orthostatic test (quiet standing for at least ten minutes). It is known that quiet standing is a severe cardiovascular stress causing a marked autonomic reaction [4]. The term "resting" in this study therefore only means "not exercising".

From experiments with pharmacological and surgical denervation of the heart it is known that the regulation of variation in heart rate is mediated through the vagal nerve [7]. From the present studies, in which the problem of the afferent pathway inherent in deep breathing experiments was avoided, it seems reasonable to conclude that the reduced variations in heart rate in diabetics are due to a progressive vagal denervation. Similarly, reduced spontaneous variation in functions controlled by other parts of the autonomic nervous system (blood flow in the foot [11] and the movements of the pupil of the eye [6]) has previously been described in long-term diabetics.
We have shown that both long-term shifts, cyclic variation, and random variation in pulse rate, measured as outlined above, are reduced in parallel in long-term diabetics. However, since the MSSD is the parameter most insensitive to systematically induced variations in pulse rate, its interpretation carries with it a minimum of assumptions concerning reproducibility of stimuli, cooperability of subject and long-term stability of the timing mechanisms in the recording and measuring equipment. Furthermore, the isolated reduction of the MSSD in diabetics with five to ten years duration might indicate that this measurement is the best for the early detection of autonomic nervous system abnormalities in diabetes.

It should be emphasized that the technique employed in this study is very simple and reproducible and only requires an ordinary ECG apparatus and a ruler.

\section{References}

1. Aagenæs, O.: Neurovascular examinations on the lower extremities in young diabetics. Thesis. Copenhagen: C. Hamburger Press 1962

2. Rundles, R.W.: Diabetic neuropathy; general review with report of 125 cases. Medicine (Baltimore) 24, 111-160 (1945)

3. Sharpey-Schafer, E.P.: Absent circulatory reflexes in diabetic neuritis. Lancet 1960 I, 559-562

4. Christensen, N.J.: Plasma catecholamines in long-term diabetics with and without neuropathy and in hypophysectomized subjects. J. Clin. Invest. 51, 779-787 (1972)

5. Neubauer, B., Christensen, N. J.: Norepinephrine, epinephrine, and dopamine contents of the cardiovascular system in long-term diabetics. Diabetes 25, 6-10 (1976)

6. Gundersen, H.J.G.: An abnormality of the central autonomic nervous system in long-term diabetes: Absence of hippus (Abstract). Diabetologia 10, 366 (1974)

7. Wheeler, T., Watkins, P.J.: Cardiac denervation in diabetes. Br. Med. J. 1973 IV, 584-586

8. Murray, A., Ewing, D. J., Campbell, I.W., Nelson, J.M.M., Clarke, B. F.: RR interval variations in young male diabetics. Br. Heart J. 37, 882-885 (1975)

9. Diem, K., Lentner, C. (Eds.): Scientific tables, pp. 193-194. Basle: J. R. Geigy F. A. 1970

10. Kendall, M.G.: Rank correlation methods, 2nd Ed. New York: Hafner Publishing Co. 1955

11. Christensen, N. J.: Spontaneous variations in resting blood flow, postischaemic peak flow and vibratory perception in feet of diabetics. Diabetologia 5, 171-178 (1969)

Received: August 20,1976, and in revised form:

December 12, 1976

Dr. H. J. G. Gundersen

Aarhus Kommunehospital

II. University Clinic of Internal Medicine

DK-8000 Aarhus C

Denmark 